\title{
Plasmon properties of multilayer albumin/gold hybrid nanoparticles
}

RECEIVED

16 August 2018

REVISED

11 September 2018

ACCEPTED FOR PUBLICATION

2 November 2018

PUBLISHED

1 February 2019

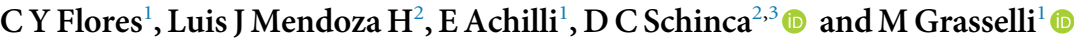 \\ 1 Laboratorio de Materiales Biotecnológicos (LaMaBio), Universidad Nacional de Quilmes-IMBICE (CONICET-CICPBA), Bernal, \\ Argentina \\ 2 Centro de Investigaciones Ópticas (CIOp), (CONICET La Plata-CIC), Gonnet, Buenos Aires, Argentina \\ 3 Departamento de Ciencias Básicas, Facultad de Ingeniería, UNLP, Argentina \\ E-mail: mariano.grasselli@unq.edu.ar
}

Keywords: multilayer protein coating, Au/Albumin nanoparticles, hybrid nanoparticles, plasmon

Supplementary material for this article is available online

\begin{abstract}
Hybrid nanoparticles are mainly based on inorganic (metal, semiconductors) and organic (proteins, polymers) compounds. The inorganic compounds are metallic nanostructures with unique features such as localized surface plasmon resonance (LSPR) bands in the UV-visible-near IR spectral range. The LSPR frequency is extremely sensitive to small changes in the physicochemical environment surrounding NPs and is dependent on NP shape and size. Recently, we reported a novel preparation method of hybrid nanoparticles based on an Albumin multilayer coating of AuNPs, stabilized by radiation-induced crosslinking processes. With this method, we obtained core/shell nanoparticles composed by Au and albumins. The aim of this paper is the description of the plasmon properties of AuNPs and other core/shell gold/protein NPs prepared. UV-vis spectra were used to detect changes of the plasmon extinction of hybrid nanoparticles, in accordance to the Mie Theory. DLS and TEM measures were used to correlate the results obtained. UV-vis spectroscopy proved to be key to characterize protein layer through two parameters: thickness and refractive index. In conclusion, UV-vis spectroscopy can be a very useful quality control methodology to prepare hybrid $\mathrm{Au}$ /protein NPs.
\end{abstract}

\section{Introduction}

Inorganic NPs have been prepared from different materials, such as metals, oxides, semiconductors and polymers; however, gold NPs (AuNPs) are one of the nanoparticle systems most abundant in the literature [1]. Since the early stages of the 'nanotechnology age', AuNPs have been recognized as an excellent scaffold for the fabrication of novel chemical and biological sensors. Given their distinct physical and chemical attributes, availability of established protocols to prepare them at different sizes [2] as well as using 'green' reductants such as tea leaves [3] or plant extracts [4], AuNP's are a widespread nanomaterial.

AuNP surface capping using organic molecules, especially those containing sulphur atoms, are very well described in the literature [5]. This property of chemical interaction has been amply used for functionalization of the surface with different biomolecules. It has been used for several decades for preparation of rapid diagnostics tests [5], and it has become one of the most studied nanomaterials for (bio)chemical sensing and diagnosis [6, 7]. An exponential number of reports have been published in the last decade on this topic. A recent review in this field covers more than a thousand scientific papers [8].

Plasmonic nanoparticles are mainly constituted by Au or Ag cores, with unique features such as localized surface plasmon resonance (LSPR) bands in the UV-visible-near IR spectral range and fluorescence quenching. The LSPR frequency is characteristic of the size and shape of nanostructure and is also extremely sensitive to small changes in the physicochemical environment surrounding NPs. Some of the features of light-matter interaction can be explained through the Mie theory, which provides the optical properties of particles size in the nanometer size range and their optical constant. It is important to take into account that the LSPR of AuNPs has also been explained by means of assembly and surface chemistry [9]. Furthermore, AuNPs display localized surface plasmon resonance (LSPR), which can be used for biosensing and dark field imaging [1]. All the optical 
properties of AuNPs will render a most relevant building block in nanostructures with two or/more functions depending on the requirements of biomedical applications.

Inorganic materials in the composition of NPs confer them attractive features such as improved biomedical diagnosis and imaging sensitivity or magneto responsiveness [10-12], which has led to a growing interest in the development of novel 'theranostic' nanotechnology devices [13]. However, nanomaterials for in vivo applications require a special surface treatment to become compatible with the human body.

Analysis of interaction of NPs with biological media, such as blood, interstitial fluid or extracellular matrix, rapidly reveals a complex interaction between NPs and the environment. Their surface is rapidly covered by macromolecules, especially proteins, with the formation of a 'corona' shell which alters the particle size, stability and in consequence, the surface properties [14]. It has been recognized that the generation of corona shells around inorganic NPs and their dynamics is a very complex process to address [15-19]. Recently a centrifugation-based physicochemical method has been proposed to study the interaction of nanoparticles with proteins without labeling [20].

Several proteins, such as Albumin, are partially modified as a consequence of the strong interaction between some chemical moieties of the protein and the highly structured surface of the AuNPs. Recently, using XANES and molecular modeling simulation, Wang et al have demonstrated that albumin 'spreads' onto the inorganic surface and exposes eight internal disulphide bridge to a flat gold surface of Au-nanorod [18, 21].

Protein corona layer has a pivotal role in the final destination of the NPs within the body on account of their interactions the local environment [22]. Therefore, the development of highly structured surface topography onto NP surface is an important issue to address in the near future. In order to reduce the nonspecific adsorption and interactions with other proteins, our goal is to prepare AuNPs covered with a stable protein multilayer coating in which macromolecules preserve their native state. To this end, we select Albumin as building block to prepare the coating layer that could achieve the objectives above.

In a recent paper, we described a novel method of the synthesis of core/shell Au/Albumin NPs by radiation induced crosslinking [23]. Plasmon spectra of these hybrid NPs showed a shift of several nanometers to longer wavelength. This observation drove us to deep insights into these phenomena in order to understand and evaluate the chance of usefulness of this UV-vis spectroscopy to analyze these changes in the protein coating. In this paper, we applied the Mie theory to describe the plasmon properties of AuNPs and other core/shell gold/ protein NPs.

\section{Materials and methods}

Gold (III) auric chloride hydrate $\left(\mathrm{HAuCl}_{4} \cdot \times \mathrm{H}_{2} \mathrm{O}\right)$ and commercial human serum albumin 20\% (HSA) was kindly donated by Laboratorio de Hemoderivados UNC. The commercial serum albumin solution has $\mathrm{N}$-acetyl tryptophan and sodium caprylate as additives and it was used as received. All other reagents were purchased from local suppliers an analytical grade and used as received.

\subsection{Synthesis of Au/Albumin nanoparticles (Au/Alb NPs)}

AuNPs were prepared according to the Frens method [24], which consists of the reduction of chloroauric acid with sodium citrate. For our assay, $100 \mathrm{ml}$ of $1 \mathrm{mM}$ chloroauric solution were boiled in a clean flask and subjected to continuous stirring. Then, $10 \mathrm{ml}$ of sodium citrate (at $39 \mathrm{mM}$ ) were added to the boiling solution and kept for one hour under continuous stirring. This synthesis was carried out in an aqueous medium to obtain a nanostructured system compatible with biological media. The NPs were stored at $4 \mathrm{C}$ in dark. The concentration of AuNPs was determined through UV-vis spectroscopy. The maximum $Q_{\text {ext }}$ of the LSPR was recorded for serials dilutions of AuNPs in aqueous solutions. Assuming a spherical shape and size for observation of TEM images, the mass of each NP was calculated from the density of ffc gold $(19,93 \mathrm{~g} / \mathrm{cm} 3)$ and Ar of atomic weight of gold $(197 \mathrm{~g} / \mathrm{mol})$. This calculation and the linear fitting of $Q_{\text {ext }}$ max of dilutions determined the initial concentration of AuNPs (assuming a complete reduction of gold salt).

In the second step, AuNPs $\left(9.81 \times 10^{11} \mathrm{NPs} / \mathrm{mL}\right)$ were dispersed in different concentrations of albumins (HSA) from 0, 2.5, 5, 10 and $15 \mathrm{mg} / \mathrm{mL}$ protein. Following that, different amounts of ethanol were added dropwise onto the protein solution, keeping the temperature at $0^{\circ} \mathrm{C}$ under constant stirring. Ethanol, Albumin and AuNPs mixtures were irradiated with gamma-rays from a 60 Co source (PISI CNEA-Ezeiza) at a dose rate $1 \mathrm{kGy} / \mathrm{h}$ and keeping sample temperature in the range of $5{ }^{\circ} \mathrm{C}-10{ }^{\circ} \mathrm{C}$ during the irradiation.

AuNPs with a monolayer of albumin are prepared with dispersion of AuNPs in a final concentration of $1 \mathrm{mg} / \mathrm{ml}$ protein. 


\subsection{Determination of size and morphology of NPs}

Particle size was determined by means of two techniques: dynamic light scattering (DLS) and transmission electronic microscopy (TEM). For DLS, the samples were measured at $25^{\circ} \mathrm{C}$ using a 90Plus/Bi-MAS particle size analyzer, with a light source of $632.8 \mathrm{~nm}$ and a $10-\mathrm{mW}$ laser. For TEM, samples were prepared by centrifugation at 14,000 rpm for $20 \mathrm{~min}$ in three cycles before measured. The measurements were carried out in Centro de Microscopias Avanzadas (CMA)-FCEyN-UBA. All measurements were carried out on days 1 and 30 after sample preparation. Atomic Force Microscopy (AFM) measures were performed with Au/cHSA NPs in Peak Force Mode. The samples were deposited in freshly cleaved mica, dried and imaging in water. Images were obtained using a Dimension Icon in Peak Force QNM (PFQNM) (Bruker $\left.{ }^{\circledR}\right)$, (Institute of Biophysics, Universidade Federal do Rio de Janeiro, Rio de Janeiro, Brazil). Rectangular silicon tip with a nominal spring constant $0.8 \mathrm{~N} / \mathrm{m}$ and with tip radius of $5 \mathrm{~nm}$ were used during the measurement.

\subsection{Experimental and theoretical determination of plasmon spectra}

Experimental determination of plasmon spectra was performed in a Shimadzu 1650PC spectrophotometer in the range 300 to $800 \mathrm{~nm}$. Experimental plasmon spectra were parameterized by plasmon peak wavelength $\left(\lambda_{p}\right)$ and wavelength at half-maximum $\left(\lambda_{m}\right)$. Theoretical determination of extinction spectra were carried out using Matlab software onto desk computer based on the general Mie theory for single and core/shell spherical NPs. The metal dielectric function entering in Mie calculations was size-corrected for those cases under $10 \mathrm{~nm}$ particle radii [25]. For the case of core/shell NPs, extinction efficiency for each particle is defined by Mendoza etal [26]:

$$
q_{e x t}=\frac{2}{k^{2} b^{2}} \sum_{n=1}^{\infty}(2 n-1) \operatorname{Re}\left\{a_{n}+b_{n}\right\}
$$

where $a_{n}$ and $b_{n}$ are Mie coefficients written in terms of Bessel spherical functions, which in turn depend on NPs radii ( $a$ and $b$ are radii parameters), $k$ are defined as $2 / \lambda$ of and the wavelength dependent relative refractive indices of core and shell materials. In the presence of naked AuNPs, the equation (1) el $2 / k^{2} \mathrm{~b}^{2}$ could be replace for $2 / k^{2} \mathrm{a}^{2}$. For metals like gold, the plasmon spectra are represented for radii of $a$ and $b$, where there are wavelength values which, for given radii $a$ and $b$, yield maximum values of $a_{n}$ and $b_{n}$ thus producing a peak in $q_{\text {ext }}$, which is known as plasmon resonance.

The previous theoretical description holds for single particle size. However, real NPs samples show a size dispersion that is most frequently described by a log-normal function, which is characterized by a modal diameter $\left(D_{m}\right)$ and a dispersion parameter $(\sigma)$ related to the distribution width. Therefore, the final theoretical extinction spectrum $\left(Q_{e x t}\right)$ is calculated as the weighted sum of $q_{\text {ext }}$ for the different finite sizes NPs that define the distribution (see figure 1).

Response Surface Plots (RSP) were prepared from theoretical spectra calculated from log-normal population parameters or shell parameters, according to the proposed core/shell models (see below).

\subsection{Electrophoretic analysis}

Polyacrylamide gel electrophoresis (SDS-PAGE) was performed with a total of $30 \mu \mathrm{l}$ of albumin and NPs diluted in deionized water and $10 \mu \mathrm{l}$ of sample buffer with $5 \%$ of 2 -mercaptoethanol, reaching a final concentration of $1.5 \mathrm{mg} / \mathrm{ml}$ of protein. Samples were heated up for $5 \mathrm{~min}$. Following that, aliquots of $18 \mu \mathrm{l}$ of the samples and protein markers were loaded on an $8 \%$ polyacrylamide gel and 5\% stacking gel. A Biopack Electrophoresis Unit was used to run the SDS-PAGE at a constant voltage of $8 \mathrm{~mA}$. Finally, the gel was stained using Comassie Blue and analyzed with Image J Gel Analyzes.

\section{Results and discussion}

Naked AuNPs are very sensitive to the changes of the environment caused by the addition of different organic solvents, $\mathrm{pH}$, ionic strength and temperature [27]. Those changes easily induce nanoparticle agglomeration so the functionalization of AuNPs is mandatory to prepare useful nanomaterials. The functionalization of the surface of AuNPs using organic molecules containing sulphur atoms or polymers is very extended in the literature $[15,28,29]$. Monolayers of proteins, such as antibodies, serum albumins and other proteins have been prepared by physisorption method and applied to disposable diagnostic devices [30, 31].

AuNPs have been prepared according to Frens method to reach NPs in the range of 20 to $30 \mathrm{~nm}$ [24]. This synthesis is carried out in an aqueous medium to avoid the presence of organic solvents or other hydrophobic ligands onto the NP surface. Supplementary figures S1(a) and S1(b) is available online at stacks.iop.org/MRX/ 6/055005/mmedia show a TEM image and the corresponding histogram of size distribution (mean diameter $23 \mathrm{~nm}$ ). Supplementary figure S1(c) plots the DLS histogram corresponding to the same sample (average 


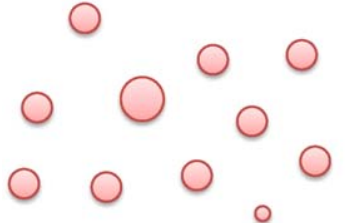

Log-normal distribution

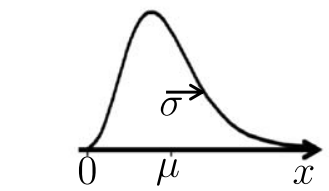

Probability $=\frac{1}{x \sigma \sqrt{2 \pi}}$

Determination of average $(\boldsymbol{\mu})$ and standard deviation $(\boldsymbol{\sigma})$ of the NP population

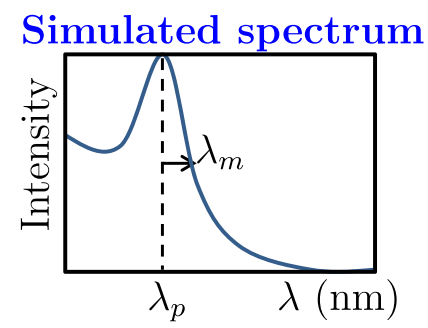

(Spherical particles)

Fitting

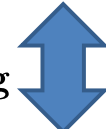

Experimental spectrum

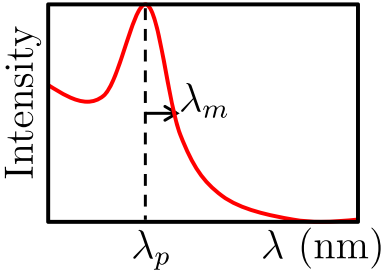

Figure 1. Scheme of work proposed. The values of $\sigma$ and $D_{m}$ of samples are determined through to comparation between theorical spectrum (in certain range of $\sigma$ and $\left.D_{m}\right)$ and experimental spectrum $\left(\lambda_{m}\right.$ and $\left.\lambda_{p}\right)$.

hydrodynamic size $33 \mathrm{~nm}$ ). The differences of size measured by both techniques are under the error dispersion of the sample, in addition to the intrinsic differences between techniques. DLS also detected a small fraction of aggregated NPs.

The experimental plasmon spectra of NPs suspensions can be characterized by two parameters: plasmon peak wavelength $\left(\lambda_{p}\right)$ and wavelength at half-maximum $\left(\lambda_{m}\right)$. Figure 2(a) shows the experimental spectrum corresponding to AuNPs prepared in this work and their parameters. Meanwhile, considering a log-normal distribution of the NP population, theoretical extinction spectrum $Q_{\text {ext }}$ is calculated as the weighted sum of the extinction efficiencies $\left(q_{\text {ext }}\right)$ calculated from equation [1]. Population distribution is characterized by a modal diameter $\left(D_{m}\right)$ and a dispersion parameter $(\sigma)$ (figure 1). A complete set of $Q_{e x t}$ were calculated for a range of $D_{m}$ and $\sigma$ and this information was plotted as a Response Surface Plot (RSP) (see figure 1). Figure 2(b) shows in a 3D graph of surface responses of $\lambda_{p}$ and $\lambda_{m}$ of the $Q_{e x t}$ as a functions of different NPs populations according to their parameters $D_{m}$ and $\sigma$. Values of $\lambda_{p}$ and $\lambda_{m}$ corresponding to the experimental spectrum shown in figure 1(a) $\left(\lambda_{p}=522 \mathrm{~nm}\right.$ and $\lambda_{m}=564 \mathrm{~nm}$ ) define two level curves in the 3D plot (figure 2(b)). The intersection of both curves (in the plane x-y) yield unique values $D_{m}=26.74 \mathrm{~nm}$ and $\sigma=0.508$. These values characterize the NP population, which matches the experimental extinction values. This data was used to calculate the $Q_{\text {ext }}$ plotted in figure 2(a) (red dashed line). It can be seen that there is a very suitable matching between experimental and theoretical curves. In figure 2(b), in addition to the level curves corresponding to the solution values of $\lambda_{p}=522 \mathrm{~nm}$ and $\lambda_{m}=564 \mathrm{~nm}$, those corresponding to $\pm 0.5 \mathrm{~nm}$ for each parameter were included as blue and black curves respectively. In these cases, intersection points differ markedly from central values, illustrating the suitability of the fitting method.

Figure 3 shows a comparison of histograms which describe the naked AuNPs population measured from TEM images (red bars) and DLS measurements (blue bars). Each histogram is fitted with a log-normal curve (red long-dashed curve and blue short-dashed lines respectively). In addition, the black full line represents the size distribution calculated from the optimal parameters fitted, according to the Mie theory and log-normal nanoparticle distribution. The calculated log-normal bell agrees with the experimental data obtained by different techniques.

\subsection{Protein coating in aqueous AuNPs suspension}

The standard preparation of HSA monolayer coating of AuNPs (Au/mHSA NPs) is performed by adding small amounts of protein to the NPs suspension [32, 33]. Albumin spreads onto the gold surface, modifying its shape $[18,21]$. Therefore, the surface exposed to the media is different from the native protein. It is our interest to prepare hybrid protein/gold NPs with a more 'native' surface resembling the Albumin surface. Our approach involves the preparation of a multilayer protein coating avoiding the surface crosslinking. Therefore, our first step will be to study the AuNPs/Albumin mixture under high protein concentrations. The system will be investigated by UV-vis spectroscopy (see figure 4 scheme of work), DLS and SDS-PAGE. 

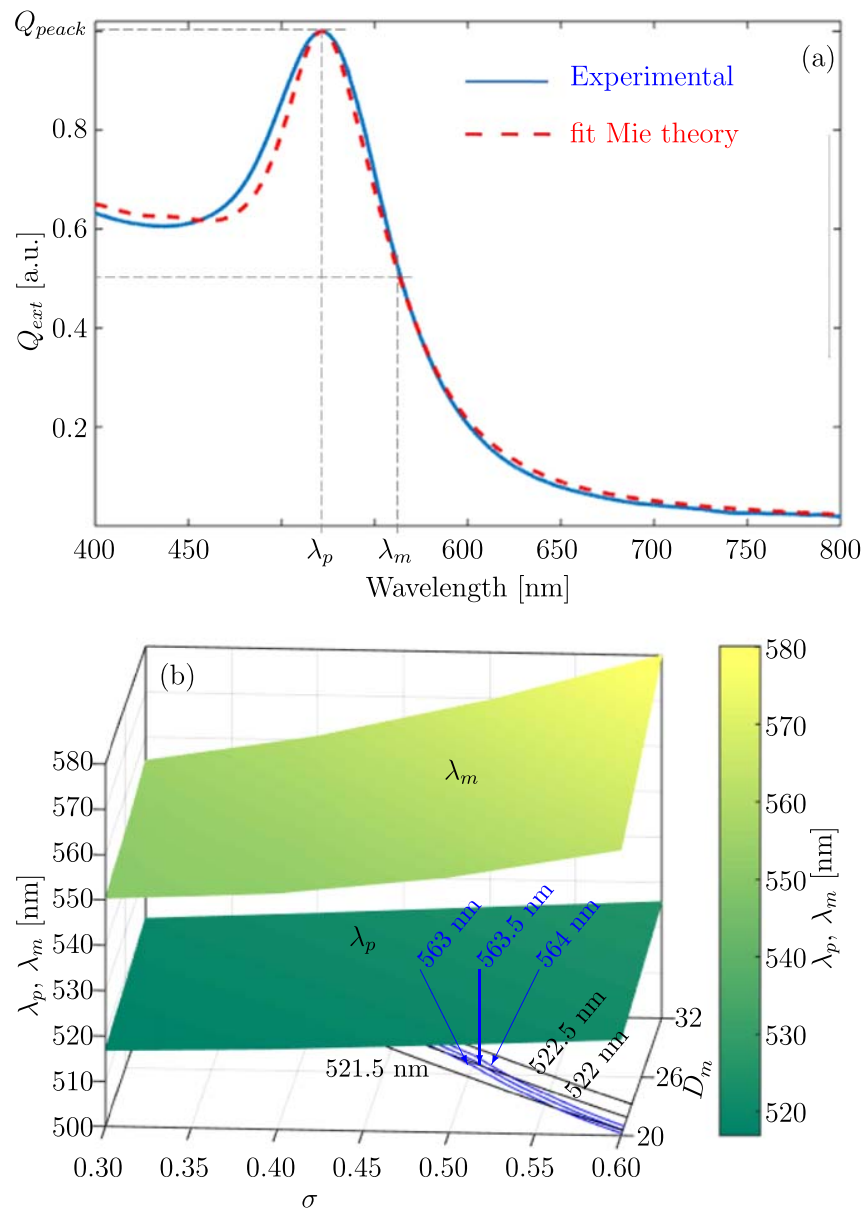

Figure 2. (a) UV-vis experimental spectrum showing the plasmon peak of AuNPs dispersed in water (blue line) and theoretical extinction spectrum with log-normal distribution of spherical AuNPs corresponding to the Mie theory (red dashed line) and (b) Response surfaces of $\lambda_{p}$ and $\lambda_{m}$ corresponding de AuNPs with differents average diameter $\left(D_{m}\right)$ and dispersion $(\sigma)$.

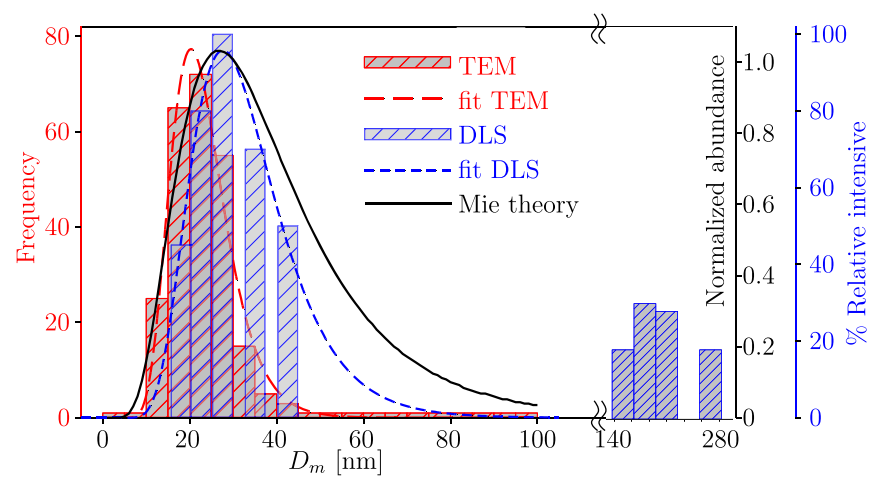

Figure 3. Histograms of AuNPs dispersion corresponding to (a) TEM images and (b) DLS measurements, (c) Plot of distributions populations AuNPs calculated by the Mie theory.

From the analysis of experimental plasmon spectra of aqueous AuNPs suspension in the presence of HSA, we detected modification of the plasmon peak position and width following increment of protein amount in the AuNPs suspension, in addition to a slight shift to higher wavelengths and changes in the peak height (figure 5(a)). As it was described in the previous section, experimental plasmon peaks are parameterized by $\lambda_{p}$ and $\lambda_{m}$ for the different HSA concentration in the media (see table 1 ).

The addition of different amounts of protein spontaneously generates core/shell NPs, of which the shell properties such as refractive index $\left(n_{c}\right)$ and thickness $(\Delta)$, are unknown. Furthermore, the alterations of the plasmon extinction are also suggests modifications in this shell structure. Figure 5(a) shows the experimental 


\section{Model 1 (Proportional layer)}

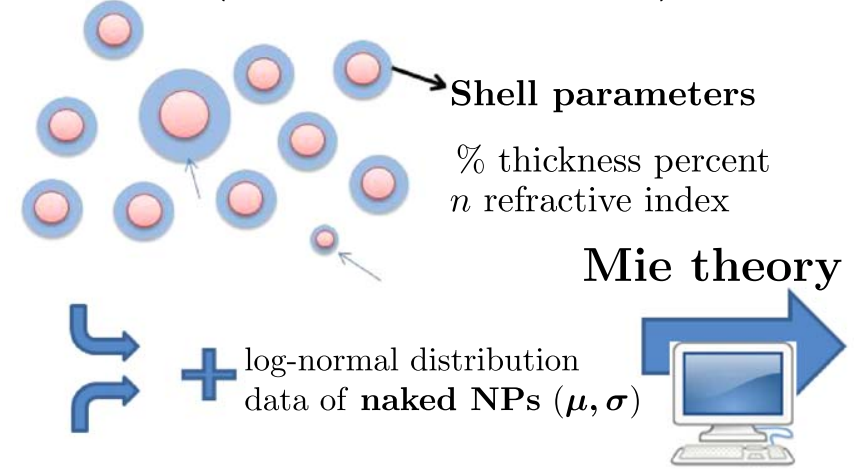

Model 2 (Steady layer)

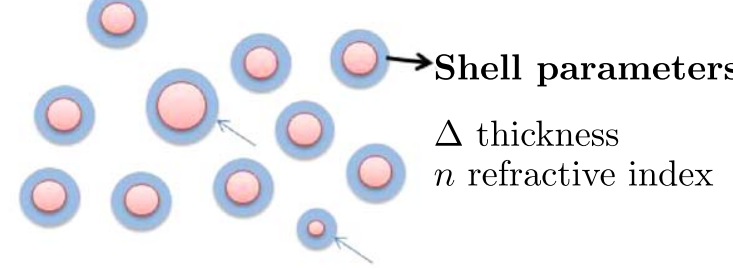

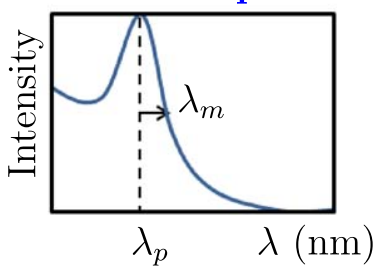

Simulated spectrum

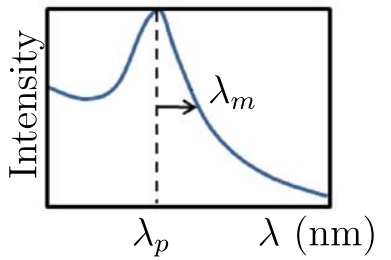

Figure 4. Scheme of models proposed for shell on surface NPs.

plasmon spectra corresponding to AuNPs suspension added with increasing amount of HSA and the variation of $\lambda_{p}$ can be seen in the insert plot. In order to explain the plasmon dispersion of the coated NPs, experimental plasmon peaks will be fitted to a core/shell NP model using the Mie theory, recently described by MendozaHerrera et al [26].

The fitting process involves the preparation of a RSP of the plasmon spectrum parameters, $\lambda_{p}$ and $\lambda_{m}$, as a function of shell parameters. The initial naked AuNPs population is defined by the log-normal distribution parameters, $D_{m}$ and $\sigma$ values. Protein shell will be defined by two new unknown parameters, $n_{c}$ and $\Delta$, which are the refractive index and thickness respectively.

The growth of protein shell will be considered according to two different coating models. See figure 5 for the two proposed models: the first approximation is a coating thickness proportional to AuNPs radii (Proportional model) and a second one in which it will be considered that all NPs will have the same coating thickness for a particular experimental condition, independent of the naked NP radii (Steady Model).

Considering both hypothesis, two RSP for $\lambda_{p}$ and $\lambda_{m}$ have been calculated for different values of $n_{c}$ and $\Delta$. In both cases, it was considered that the initial population of naked AuNPs are described by the values $D_{m}=26.74 \mathrm{~nm}$ and $\sigma=0.508$. Figure 5(b) shows the SRP of $\lambda_{p}$ and $\lambda_{m}$ corresponding to different values of $n_{c}$ and $\Delta$ of protein shell, in this case considered as a percentage of NP diameters (Model 1). In the same way, figure 5(c) shows the response surface for the second model where plasmon dispersion parameters are plotted as a function of $n_{c}$ and $\Delta$. Additionally, both figures show curves (onto the $\mathrm{x}-\mathrm{y}$ plane) corresponding to solution values of $\lambda_{p}=523.1 \mathrm{~nm}$ and $\lambda_{m}=566.5 \mathrm{~nm}$ and those corresponding to $\pm 0.5 \mathrm{~nm}$ for each parameter were included as blue and orange curves respectively. These $\lambda_{p}$ and $\lambda_{m}$ values correspond to AuNPs population in a media containing $5 \mathrm{mg} / \mathrm{mL}$ of HSA. The Model 1 does not show a theoretical solution from the curves described in figure 5(b). Only figure 5(c) presents a solution (crossing lines) to the theoretical plasmon spectrum considering a single thickness values. The simulation reaches a solution for $n_{c}=1.345$ and $\Delta=4.22 \mathrm{~nm}$. All $n_{c}$ and $\Delta$ values corresponding to the solutions using different HSA concentrations are described in table 1. Considering the experimental values which characterize the plasmon peaks, the simulation describes an increment in $\Delta$ and a relative constant $n_{c}$ value. It is to be expected therefore, that increases in the protein concentration media will lead to increments in the corona protein layer.

DLS analyzes of the samples were performed in order to find out additional experimental data to support these predictions. As a consequence of the important differences in the optical properties of both components (inorganic and organic), the light dispersion data of DLS was collected as \% Intensity instead of \% Relative number. In the analysis of multimodal samples, the former one does not keep the proportionality between populations of different sizes. However, it has been considered for a qualitative analysis as a consequence of bigger-sized particle populations exponentially overvalued the intensity of the dispersed light. 

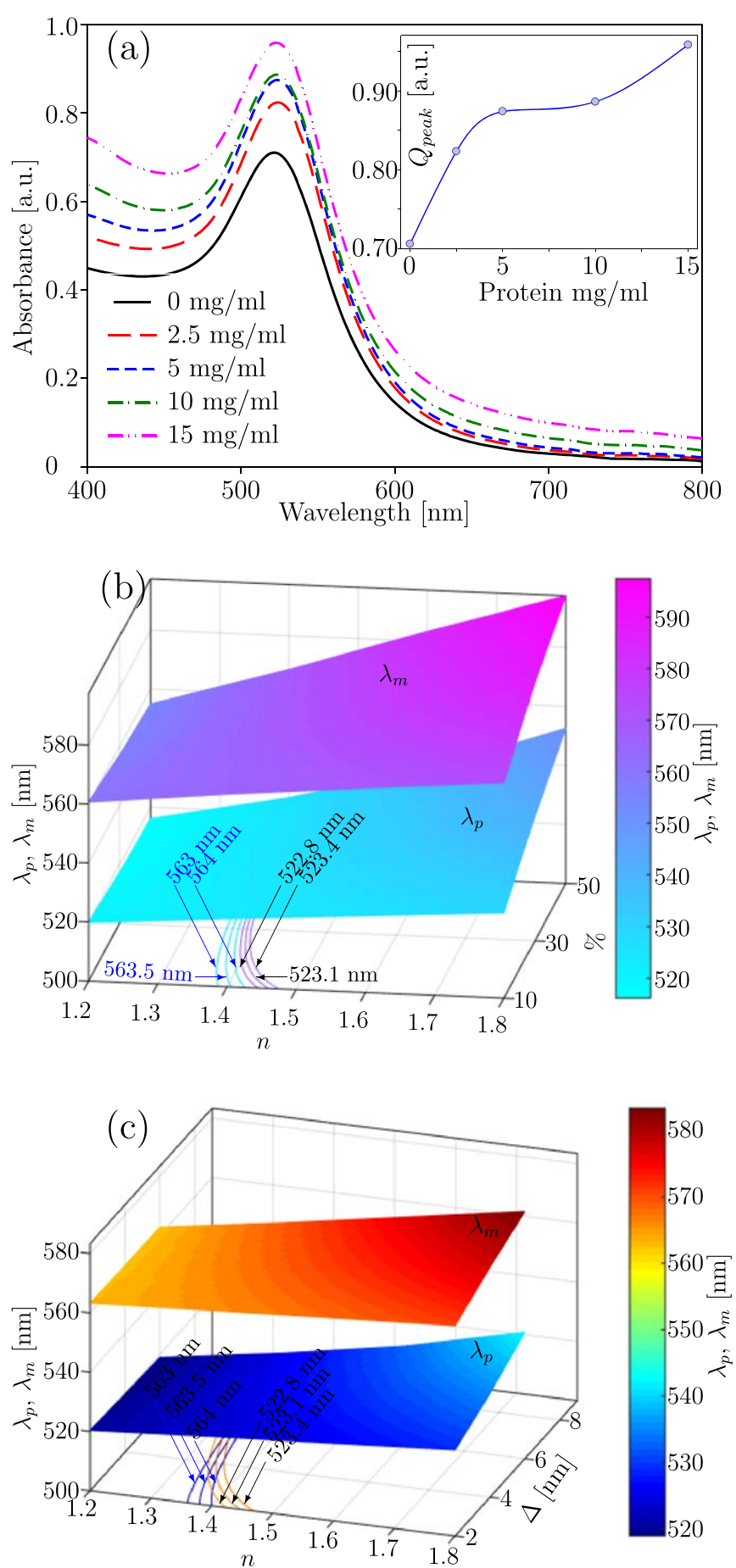

Figure 5. (a) UV-vis experimental spectrum showing the plasmon peak of AuNPs dispersed in different solutions of HSA ( $0,2.5,5,10$ y $15 \mathrm{mg} / \mathrm{ml}$ albumin) are corresponded. The inset shows the Peak intensity versus HSA concentration. All samples are in water as solvent (b) Response surfaces of $\lambda_{p}$ and $\lambda_{m}$ corresponding de AuNPs with differents refractive index $(n)$ versus $\%$ of NPs diameters corresponding at first hypothesis and (c) refraction index $(n)$ versus thickness $(\Delta)$ corresponding at second hypothesis. In both cases in the presence of $5 \mathrm{mg} / \mathrm{ml} \mathrm{HSA}$.

All histograms corresponding to DLS measurements of AuNPs in presence of HSA at high concentrations have shown three main particle populations (figure 6). Those consigned to free $\mathrm{BSA}\left(R_{H}\right.$ is $3.4 \mathrm{~nm}$, [34]) or small size albumin aggregations $\left(R_{H}<6 \mathrm{~nm}\right)$; AuNPs coated by albumins; and aggregates. The intermediate size population has an average hydrodynamic size of approximately $44 \pm 9.7 \mathrm{~nm}$, assigned to a single AuNPs covered with HSA. The relative proportion of each population cannot be determined; however, the monolayer coated AuNPs are present in the aqueous systems and a minor amount of aggregates can be estimated.

The experimental data corresponding to particle size in solution is far more complicated than the predicted values. However, simulation would support the understanding of the main aggregation process involved. The differences in the $\Delta$ values predicted by the fitting data from the plasmon peak are smaller than the error in the 

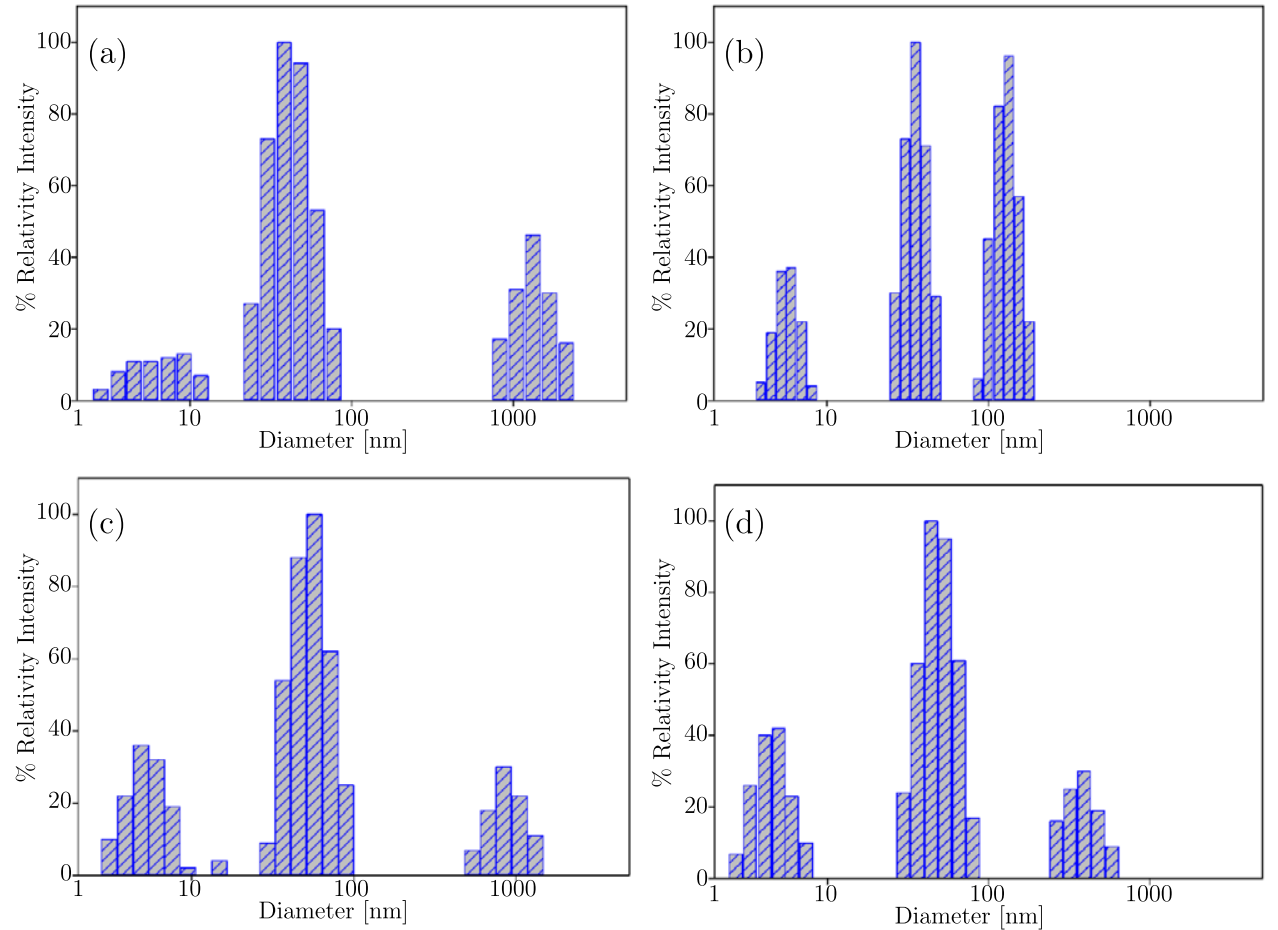

Figure 6. DLS histograms (Intensity \% versus diameter size) corresponding to AuNPs in aqueous solution with the addition of $2.5 \mathrm{mg} / \mathrm{ml} \mathrm{HSA}$ (top left); $5 \mathrm{mg} / \mathrm{ml}$ (top right); $10 \mathrm{mg} / \mathrm{ml}$ (bottom left) and $15 \mathrm{mg} / \mathrm{ml}$ (botton right).

\begin{tabular}{|c|c|c|c|c|c|c|}
\hline$[\mathrm{mg} / \mathrm{ml}]$ & $\lambda_{p}$ & $\lambda_{m}$ & $D_{m}$ & $\sigma$ & $n_{c}$ & $\Delta$ \\
\hline 0 & 522 & 564 & 26.74 & 0.508 & & \\
\hline 2.5 & 524.5 & 566.5 & 26.74 & 0.508 & 1.346 & 3.03 \\
\hline 5 & 523.1 & 566.5 & 26.74 & 0.508 & 1.345 & 4.22 \\
\hline 10 & 523.5 & 567.5 & 26.74 & 0.508 & 1.345 & 6.05 \\
\hline 15 & 523.5 & 569.5 & 26.74 & 0.508 & 1.346 & 8.07 \\
\hline
\end{tabular}

DLS data, therefore it cannot used to confirm the predictions. However, the model has been useful to discard the coating process in which the shell thickness is proportional to the NP size (Model 1).

\subsection{Plasmon properties of aqueous-ethanol AuNPs suspensions}

Low proportions of ethanol in water have a minor effect on AuNPs dispersion. The polarity of R-OH can play the role of the ionic double layer to keep the NP dispersion relatively stable, although not as well as water does [35]. The experimental plasmon peak shifts to high wavelength can be measured only for a solution containing $40 \%$ ethanol or higher (figure 7 and table 2).

Taking the Mie theory account, the small shift measured for AuNPs dispersed in ethanol/water mixture can be explained by changes in the $n_{c}$ of the surrounding media. From the literature, the $n_{c}$ for ethanol/water mixtures has the following values $1.3439,1.3496,1.3543$ and 1.3580 for $10,20,30$ and $40 \%$ ethanol respectively [36]. Considering a density of $0.789 \mathrm{~g} / \mathrm{mL}$ for the absolute ethanol, the mass concentration will be $0.1578,0.2367,0.3156$ and 0.3945 for these solutions respectively. From the fitting process, as described in figure 2(b), we determined a shifting of the $\lambda_{p}$ to higher wavelengths with the increment of the $n_{c}$ as a consequence of the ethanol concentration increase. However, these increments are lower than $1 \mathrm{~nm}$ and it is experimentally relevant at ethanol concentrations higher than $40 \%$.

\subsection{Protein coating in aqueous-ethanol AuNPs suspension}

The ternary mixture prepared with albumin, ethanol and water is studied considering $30 \%$ of ethanol in the mixture. Under this experimental condition, the solvent will not disturb the plasmon peak. Therefore, 


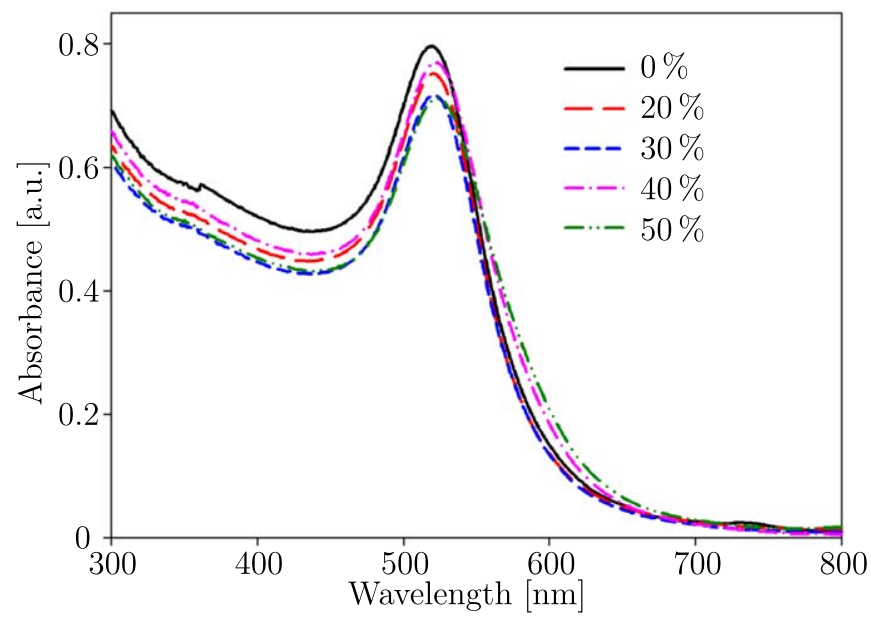

Figure 7. UV-vis spectra showing the plasmon peak of AuNPs in water following the addition of different amounts of ethanol from $0 \%$ to $50 \% \mathrm{v} / \mathrm{v}$.

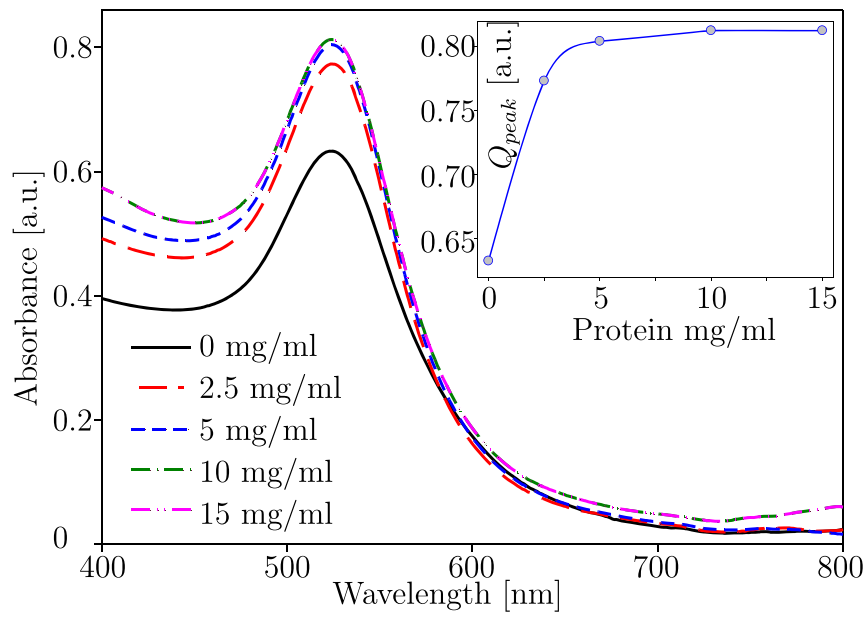

Figure 8. UV-vis spectra showing the plasmon peak of Au/HSA NPs prepared with initial HSA concentrations of 2.5, 5, 10 and $15 \mathrm{mg} / \mathrm{ml}$ in ethanolic solutions at $30 \% \mathrm{v} / \mathrm{v}$. The insert shows the Peak intensity versus HSA concentration. All samples are in water as solvent.

Table 2. Experimental data are corresponding at the plasmon peak of AuNPs in different proportions of ethanol in the media.

\begin{tabular}{lcc}
\hline$\% \mathrm{v} / \mathrm{v} \mathrm{EtOH}$ & $\lambda_{p}$ & $A b s_{p}$ \\
\hline 0 & 520 & 0.796 \\
20 & 520 & 0.752 \\
30 & 520 & 0.717 \\
40 & 523 & 0.769 \\
50 & 524 & 0.712 \\
\hline
\end{tabular}

increasing concentrations of albumin from 2.5 to $15 \mathrm{mg} / \mathrm{ml}$ into the AuNPs suspension, prepared in aqueous ethanol media, were followed by visible spectrophotometry (figure 8). Experimentally, we found a slight shift of the AuNP plasmon peak to higher wavelengths and increased intensity, with the increase of protein concentration (see figure 8 insert). This behavior, which could be associated to the interaction of protein molecules with the gold surface, is independent of the solvent composition. Tsai et al reported an increase of 

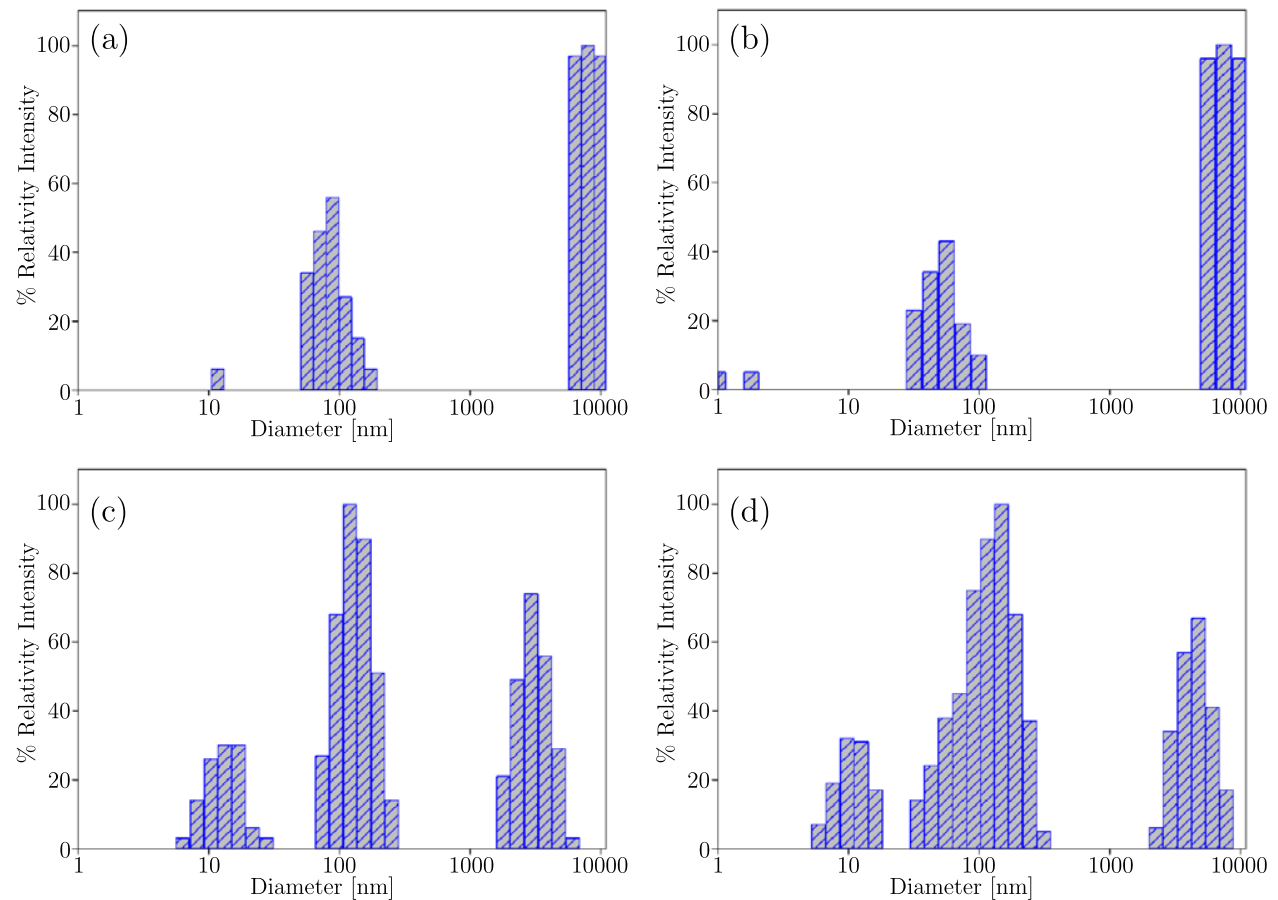

Figure 9. DLS histograms (Intensity \% versus diameter size) corresponds to AuNPs in aqueous ethanol solution ( $30 \% \mathrm{v} / \mathrm{v})$ with the addition of $2.5 \mathrm{mg} / \mathrm{ml} \mathrm{HSA}$ (top left); $5 \mathrm{mg} / \mathrm{ml}$ (top right); $10 \mathrm{mg} / \mathrm{ml}$ (bottom left) and $15 \mathrm{mg} / \mathrm{ml}$ (botton right).

Table 3. Experimental and fitted

data are corresponding to the

extintion spectra of AuNPs in aqueous ethanol solution $(30 \%$ $\mathrm{v} / \mathrm{v}$ ) with the addition of $2.5,5$

10 and $15 \mathrm{mg} / \mathrm{mlHSA}$. The experimental data $\lambda_{p}$ and $\lambda_{m}$ characterize the extintion.

\begin{tabular}{lcc}
\hline$[\mathrm{mg} / \mathrm{ml}]$ & $\lambda_{p}$ & $\lambda_{m}$ \\
\hline 0 & 524.5 & 571 \\
2.5 & 524 & 566 \\
5 & 524 & 566.5 \\
10 & 524.5 & 567.5 \\
15 & 524.5 & 567.5 \\
\hline
\end{tabular}

plasmon intensity associated to an electrostatic interaction between them [37]. Also, it was observed, in both cases, the intensity of plasmon peak reaches a plate value from $5 \mathrm{mg} / \mathrm{ml}$ of albumin. Under these experimental conditions the $\mathrm{n}_{\mathrm{c}}$ of the ethanol/water solution ( $30 \%$ ethanol) is comparable to the $n_{c}$ of the coated protein layer $\left(n_{c}(\right.$ ethanol $30 \%)=1.3543$ versus $n_{c}($ AuNPs/albumin $\left.)=1.345\right)($ see table 3$)$, therefore the plasmon spectra is similar for different protein amounts (similar values of $\lambda_{p}$ and $\lambda_{m}$ ).

However, DLS analysis shows a completely different scenario for these samples (figure 9). The most important characteristic of these histograms is the absence of the free albumin population and low HSA concentrations do not have particles lower than $30 \mathrm{~nm}$ diameter. There are records of samples with 10 and $15 \mathrm{mg} / \mathrm{ml} \mathrm{HSA}$, with a particle population in the range of 6 to $20 \mathrm{~nm}$, which can be assigned to an aggregation of only albumin molecules. This phenomenon has been recently described for BSA molecules in aqueous ethanol solution [38]. Albumin aggregation can generate small aggregates whose sizes have a direct correlation with the ethanol concentration [38].

The existence of high MW aggregates in the low-concentration samples can be explained by the presence of AuNPs aggregates where there are not enough albumin molecules to produce stable multilayer coating of AuNPs. Meanwhile, samples containing high albumin concentrations show aggregates of much lower MW than the low-concentration ones and in much lower proportion than the medium-size populations. 
Table 4. Hydrodynamic diameter of AuNPs/HSA aggregates in water and aqueous ethanol solution $(30 \% \mathrm{v} / \mathrm{v})$ determined by DLS in the presence of different amounts of protein.

\begin{tabular}{lcc}
\hline $\begin{array}{l}\text { Concentration of } \\
\text { albumin }(\mathrm{mg} / \mathrm{ml})\end{array}$ & $\begin{array}{c}\mathrm{DH}(\mathrm{nm}) \text { in water } \\
\text { non-irradiated }\end{array}$ & $\begin{array}{c}\mathrm{DH}(\mathrm{nm}) \text { in } 30 \% \mathrm{~V} / \mathrm{V} \\
\text { EtOH non-irradiated }\end{array}$ \\
\hline 2.5 & 38 & $>5000$ \\
5 & 35 & $>5000$ \\
10 & 57 & 149 \\
15 & 44 & 120 \\
\hline
\end{tabular}

Finally, the medium-size particles population has a diameter shifting from aggregates size lower than $100 \mathrm{~nm}$ to higher than $100 \mathrm{~nm}$ when the HSA increases from $2.5 \mathrm{mg} / \mathrm{ml}$ to $15 \mathrm{mg} / \mathrm{ml}$. Meanwhile, the DLS histogram corresponding to $10 \mathrm{mg} / \mathrm{ml}$ suggests the presence of two medium-size populations. Table 4 summarizes the average diameters of these populations for the studied conditions. In relation to the sizes, these populations are compatible with NPs containing one AuNP core per particle and increasing amount of albumins in the shell when higher concentration of HSA and ethanol are present in the solution. This assumption can be supported by the simulation findings corresponding to the fact at higher protein concentration, higher shell thickness.

Considering the dynamic aggregation of albumins in aqueous ethanol solutions [38], it is to be expected that the hybrid Au/HSA NPs will be unstable in a media without ethanol. Therefore, a crosslinking process is mandatory to stabilize the suspension.

\subsection{Protein shell crosslinking}

Several chemical and physical treatments have been reported for preparation of albumin micro and nanoparticles [39, 40]. Regarding the physical treatments, our research group has developed a novel crosslinking method of proteins based on ionizing radiation [41]. Gamma rays are ionizing radiation that can induce chemical changing in the irradiated materials, where crosslinking and degradation effects onto the soft mater are achieved. The preponderance of either of the two effects depends on the chemistry of macromolecules, the physical state of the matter and the irradiation conditions. In an aqueous environment, the main effect is degradation; however, a high concentration of ethanol $(>6 \mathrm{M})$ and irradiation conditions used in this work (low dose rate) reduce this effect as a consequence of avoiding the generation of hydroxyl radicals and hydrogen peroxide in the medium [42]. Therefore, a preponderance of crosslinking can be achieved. Additionally, the crosslinking process could be intermolecular or intramolecular depending on the tuning of the experimental conditions [43].

Recent studies have focused on irradiation in a gamma source of the ternary mixture of AuNPs, albumin in a mixture of ethanol and water [23]. Spherical shape Au/albumin NPs of average diameter of about $60 \mathrm{~nm}$ was prepared with a core/shell structure. Only one kGy irradiation dose was enough to show changes in the plasmon peak characteristics. In addition, changes in the protein shell stability were shown by reduction in the dissolution rate of gold core under cyanate solutions [23].

figure 10 shows the plasmon peak of three different $\mathrm{Au} /$ albumin NPs preparations. The standard $\mathrm{Au} / \mathrm{mHSA}$ NPs in aqueous solution; multilayer albumin coated AuNPs on 30\% v/v ethanol (Au/HSA NPs) and the radioinduced crosslinking one (Au/cHSA NPs) dispersed in aqueous solution. Au/cHSA NPs have a plasmon peak shift to higher wavelengths with respect to Au/HSA NPs and Au/mHSA NPs samples. The plasmon shift wavelength was $10 \mathrm{~nm}$ approximately, in addition to an increment of the peak intensity. In the case of multilayer coated NP (Au/HSA NPs) in ethanol/water mixture, we did not observe any change in the plasmon peak (see above). Table 5 depicts the data fitted ( $\Delta$ and $n_{c}$ ) from the spectra of the figure $10\left(\lambda_{p}\right.$ and $\left.\lambda_{m}\right)$ using the model described previously according to the Mie theory. In relation to the output fitted values, NPs coated with albumin without irradiation reached the same refractive index and have shown a significant shell thickness difference (longer ones in the presence of ethanol). Meanwhile, the irradiated sample shows an increase in both parameters. In the case of refractive index, the increment could be due to changing the chemical structure of the protein as a consequence of the ionizing radiation (table 5).

Considering the amount of initial protein concentration in the preparation of Au/cHSA NPs, the plasmon signal of the irradiated samples shows a maximum at $2.5 \mathrm{mg} / \mathrm{ml}$ of HSA in the suspension (Supplementary figure S2). This behavior is different to that determined for the non-irradiated samples. The irradiation process disrupts the dynamic aggregation of proteins onto NPs surface in the presence of ethanol, as a consequence of a crosslinking process induced by the reductive radicals from the solvent radiolysis, as it was described previously [38]. 


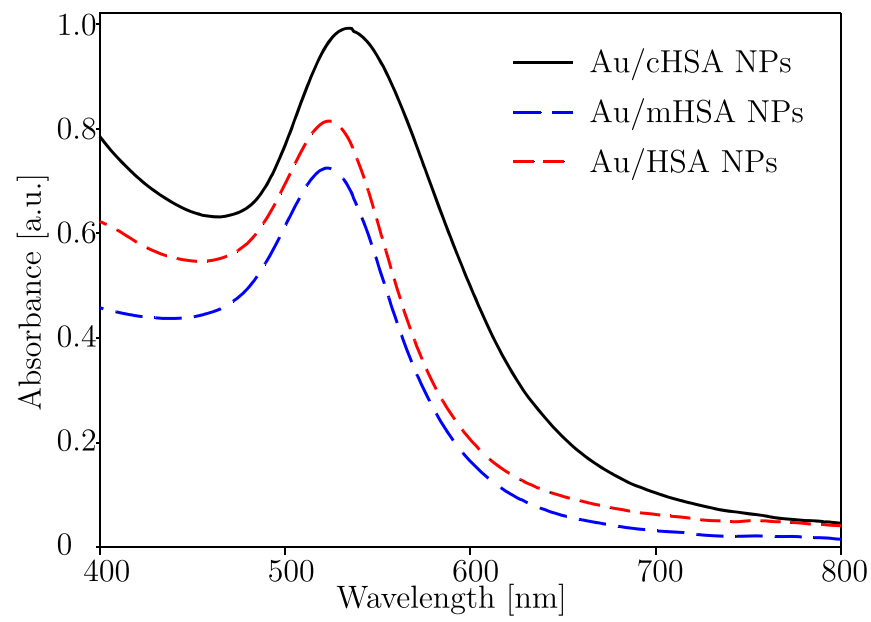

Figure 10. UV-vis spectra showing the plasmon peak of AuNPs covered with a monolayer of HSA prepared by a standard method (Au/mHSA NPs); Au/HSA NPs with a multilayer coating without crosslinking (non-irradiated) and stabilized by radiation-induced crosslinking (Au/cHSA NPs). All samples are in water as solvent.
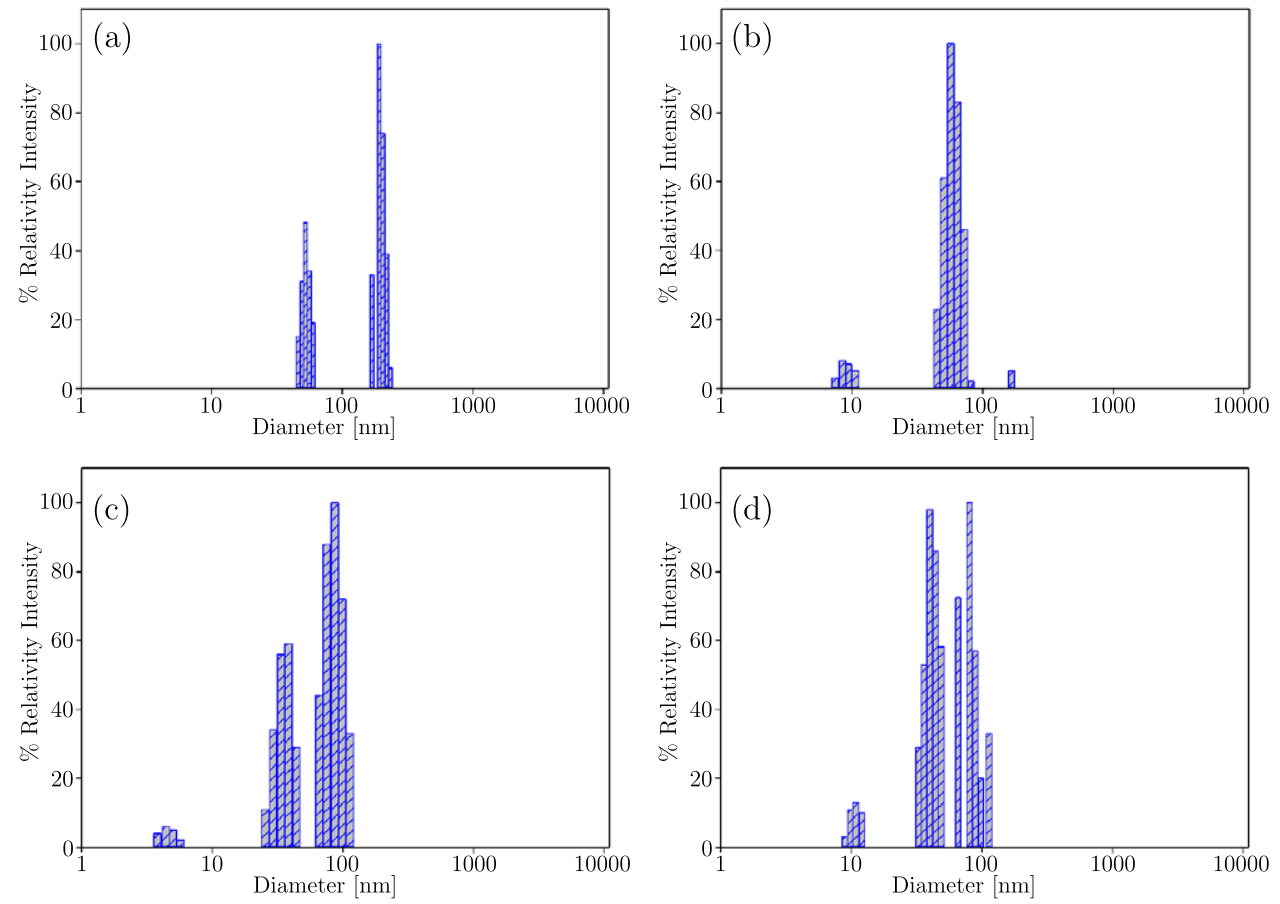

Figure 11. DLS histograms (Intensity \% versus diameter) corresponding to $\mathrm{Au} / \mathrm{cHSA}-\mathrm{NPs}$ in water. These samples were prepared in aqueous ethanol solution $30 \% \mathrm{v} / \mathrm{v}$ with the addition of $2.5 \mathrm{mg} / \mathrm{ml} \mathrm{HSA}$ (top left); $5 \mathrm{mg} / \mathrm{ml}$ (top right); $10 \mathrm{mg} / \mathrm{ml}$ (bottom left) and $15 \mathrm{mg} / \mathrm{ml}$ (botton right).

Table 5. Experimental and fitted data corresponds to the extintion spectra of Au/mHSA NPs (monolayer coated); Au/HSA NPs (multilayer coated without crosslinking (non-irradiated)) and stabilized by radiationinduced crosslinking (Au/cHSA NPs). The experimental data $\lambda_{p}$ and $\lambda_{m}$ characterize the extintion spectra and $n_{c}$ (refractive index) and $\Delta$ the calculated protein layer.

\begin{tabular}{lccccccc}
\hline NPs & Coating & Solvent & Irradiation $^{1}$ & $\lambda_{p}$ & $\lambda_{m}$ & $D_{m}(\mathrm{~nm})$ & $n_{c}$ \\
\hline $\mathrm{Au} / \mathrm{mHSA}$ & Monolayer & Water & No & 523 & 567.5 & 6.3 & 1.34 \\
$\mathrm{Au} / \mathrm{HSA}$ & Multilayer & Ethanol & No & 523.5 & 569 & 8.5 & 1.34 \\
& & /Water & & & & & \\
$\mathrm{Au} / \mathrm{cHSA}$ & Multilayer & Water & Yes & 532 & 587.0 & 10.7 & 1.45 \\
\hline
\end{tabular}




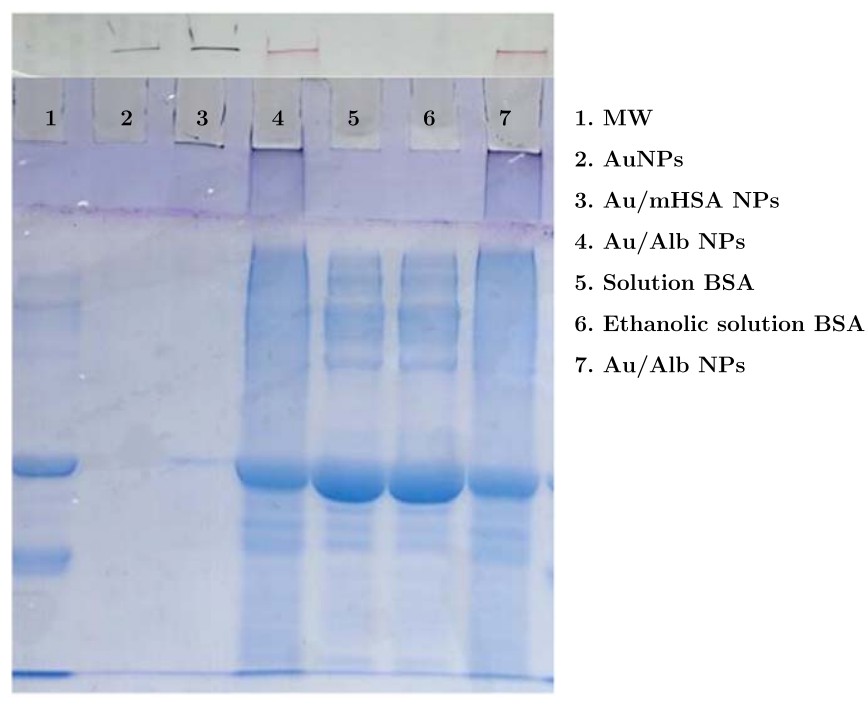

Figure 12. SDS-PAGE electrophoresis gel $8 \%$ was stained with Coomassie-dye images. The samples runned in the following order left to right:(1)molecular weight (MW), (2) AuNPs, (3)Au/mHSA NPs, (4)Au/Alb NPs, (5) solution BSA, (6)ethanolic solution of BSA and (7)Au/Alb NPs. The concentrations of Au/Alb NPs was $15 \mathrm{mg} / \mathrm{ml}$ of albumin.

The Au/cAlb NPs size was determined in aqueous solution by DLS. Considering the representation of DLS, the scattered intensity has been chosen on account of the heterogeneity of the different particles present in the system, some of which are pure protein and others have different ratios of protein and gold. Therefore, in order to avoid additional uncertainties of peak areas, the most straightforward variable was selected. Figure 11 shows the DLS histograms corresponding to systems prepared with HSA at 2.5, 5, 10 and $15 \mathrm{mg} / \mathrm{ml}$. In most of the cases, two main populations were found. The average size of Au/cAlb NPs at $2.5 \mathrm{mg} / \mathrm{mL}$ is $52 \mathrm{~nm}$. In other concentrations, the NPs size was slightly raised accordingly to increase albumin concentration.

Those results confirmed the presence of albumins multilayer to generate an increase of NP size as it was found for $\mathrm{Au} / \mathrm{HSA} \mathrm{NPs}$ in ethanol water mixture (figure 9). Histograms at concentrations from $5 \mathrm{mg} / \mathrm{ml}$ to $15 \mathrm{mg} / \mathrm{ml}$ show the presence of albumins aggregates and/or nanoparticles (Alb NPs) and Au/albumin NPs aggregates. These results were confirmed by purification of NP samples by centrifugation and Size Exclusion Chromatography.

The presence of covalent protein crosslinking was evaluated by SDS-PAGE. Different NP samples were run in $8 \%$ acrylamide gel (2.5\% stacking). Figure 12 shows the direct and Coomassie-dyed images of the stacking zone and the Coomassie-dyed running gel. Most of the AuNPs kept in seeding slots and naked and monolayer coated ones shift the color from red to blue (aggregation effect). The only exception is the Au/cHSA, which keeps its initial color. Albumin from Au/mHSA NPs runs in the gel as the HSA standard sample, and in the case of $\mathrm{Au} / \mathrm{cHSA}$ NPs, a spread of proteins was found in the range of the high molecular weight. These multiple and random crosslinking effect found in the gel could be compatible with a non-specific radiation-induced crosslinking process. A densitometry analysis of the line corresponding to $\mathrm{Au} / \mathrm{cHSA}$ NPs reveals a $34 \%$ of the protein is cross-linked.

\subsection{Microscopy analysis}

The NP shape of core/shell distribution of these NPs was determined by TEM. Proteins were visualized by staining with uranyl acetate. Figure 13 shows TEM image and its corresponding frequency histogram of $\mathrm{Au} / \mathrm{cHSA}$ NPs. The presence of a halo around black points assigned to the albumin multilayer shell is found in all NPs. The average of NPs sizes determined from TEM data were $50 \mathrm{~nm}$ for Au/cHSA NPs. Taking into account the AuNPs average size of $33 \mathrm{~nm}$, we speculated that one or two fold-size increase were due to one or two albumin layers.

An AFM image capture in solution shows a 3D shape of the protein shell. Figure 14 shows different kinds of structures in the plot: small tiny structures which are attributed to albumin (and small albumin aggregates); medium size spheres corresponding to AuNPs displaying very smooth surface and the big spheres attributed to the $\mathrm{Au} / \mathrm{cHSA}-\mathrm{NPs}$. The latter have irregular surfaces which are assigned to the heterogeneity of the crosslinking process and the amount of protein in the shell. 

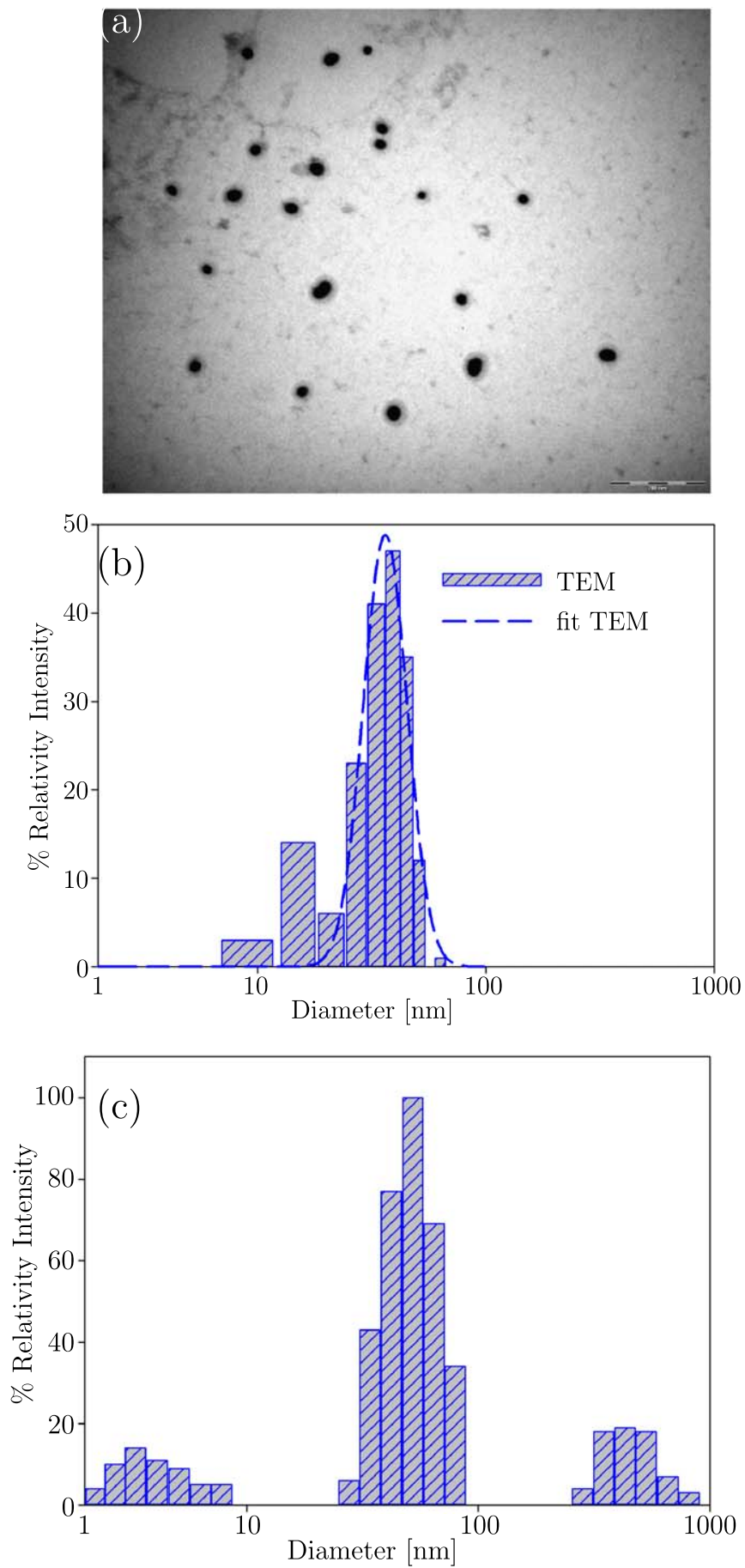

Figure 13. a) TEM picture of Au/cHSA-NPs by negative tincion with uranil acetate; (b) Histogram corresponding to the TEM image; (c) LDS histogram of the same sample in water. The concentration of HSA in samples was $15 \mathrm{mg} / \mathrm{ml}$ of protein. This samples was purified by centrifugation before at measured.

\section{Conclusions}

Plasmon extinction spectra of AuNPs under different experimental conditions have been properly fitted using the Mie theory and a log-normal distribution of the NPs population. We have used a model of a single spherical NP in a surrounding media or a single NP coated with a layer of a soft material (protein layer) in aqueous or aqueous/ethanol solutions. The protein layer has been characterized by two parameters, thickness and refractive index under the assumption that the NP has a spherical shape. The theoretical results arising from the fitting process of naked AuNPs have an excellent correlation with TEM, DLS data in addition to other data available from literature. In accordance to the core/shell simulations, the fitting process was able to discriminate between 


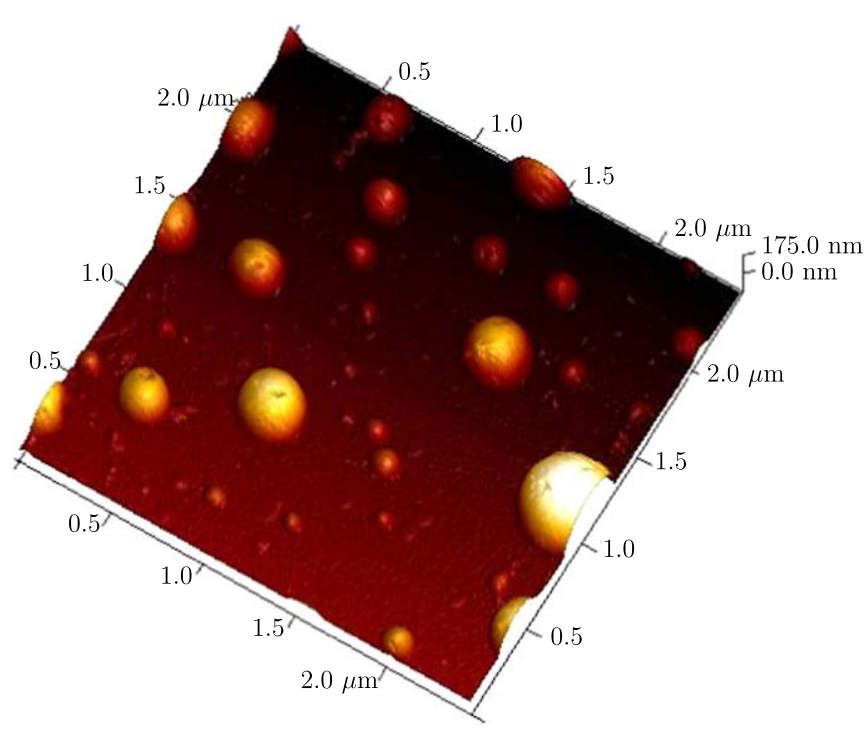

Figure 14. Image in Liquid AFM of Au/cHSA NPs. The concentration of HSA in samples was $15 \mathrm{mg} / \mathrm{ml}$ of protein. The measured are performed in aqueous solutions at room temperature.

different coating models and explain different experimental conditions. The Steady Model fits with the experimental plasmon spectrum of hybrid NPs and is also in accordance with our hypothesis about protein aggregation around AuNPs in the presence of ethanol [38].

As predicted by the experimental and simulations data described in this work, UV-visible spectroscopy is a very useful technique to characterize the hybrid NPs and could be proposed as a quality control methodology to prepare these nanomaterials.

Additional characterization of the hybrid NPs has been extended by TEM and AFM microscopy and electrophoresis, confirming the core/shell structure of these novel nanoparticles.

\section{Acknowledgments}

CYF and JMH thank CONICET for the fellowship. MG and DS are researchers from CONICET. J Montes de Oca and R Candal allowed us the DLS measures. B F de Carvalho Patricio and G Weismüller allowed us AFM images. This work was partially supported by grants from Universidad Nacional de Quilmes (Buenos Aires, Argentina), IAEA and MINCyT (Argentina).

\section{ORCID iDs}

D C Schinca (i) https:// orcid.org/0000-0001-9168-867X

M Grasselli (i) https://orcid.org/0000-0003-0927-5943

\section{References}

[1] Giner-Casares J J, Henriksen-Lacey M, Coronado-Puchau M and Liz-Marzán L M 2016 Inorganic nanoparticles for biomedicine: where materials scientists meet medical research Mater. Today 19 19-28

[2] Grzelczak M, Perez-Juste J, Mulvaney P and Liz-Marzan L M 2008 Shape control in gold nanoparticle synthesis Chem. Soc. Rev. 37 $1783-91$

[3] Nune S K, Chanda N, Katti K, Shukla R, Kulkarni R R, Thilakavathy S, S Mekapothula, Raghuraman K and Katti K V 2009 Green nanotechnology from tea: phytochemicals in tea as building blocks for production of biocompatible gold nanoparticles J. Mater. Chem. $192912-20$

[4] Song J Y, Jang H-K and SooKim B 2009 Biological synthesis of gold nanoparticles using magnolia kobus and diopyros kaki leaf extracts Process Biochemistry $441133-8$

[5] Giljohann D A, Seferos D S, Daniel W L, Patel P C, Massich M D and Mirkin C A 2010 Gold nanoparticles for biology and medicine Angewandte Chemie International Edition 49 3280-94

[6] Saha K, Agasti S S, Kim C, Li X and Rotello V M 2012 Gold nanoparticles in chemical and biological sensing Chem. Rev. 112 2739-79

[7] Yeh Y C, Creran B and Rotello V M 2012 Gold nanoparticles: preparation, properties, and applications in bionanotechnology Nanoscale 4 1871-80 
[8] Alhareth K, Sancey L, Tsapis N and Mignet N 2017 How should we plan the future of nanomedicine for cancer diagnosis and therapy? International Journal of Pharmaceutics 532 1871-80

[9] Amendola V, Pilot R, Frasconi M, Maragó O M and Iatí M A 2017 Surface plasmon resonance in gold nanoparticles: a review J. Phys.: Condens. Matter 29203002

[10] Dykmana L and Khlebtsov N 2012 Gold nanoparticles in biomedical applications: recent advances and perspectives Chem. Soc. Rev. 41 2256-82

[11] Louis C and Pluchery O 2011 Gold nanoparticles for physics, chemistry and biology (London: Imperial College Press) (https://doi.org/ $10.1142 / \mathrm{p} 815)$

[12] Li F, Lu J, Kong X, Hyeon T and Ling D 2017 Dynamic nanoparticle assemblies for biomedical applications Adv. Mater. 29 1605897

[13] Janib S M, Moses A S and MacKay J A 2010 Imaging and drug delivery using theranostic nanoparticles Adv. Drug. Deliv. Rev. 62 1052-63

[14] Cedervall T, Lynch I, Lindman S, Berggard T, Thulin E, Nilsson H, Dawson K A and Linse S 2007 Understanding the nanoparticleprotein corona using methods to quantify exchange rates and affinities of proteins for nanoparticles Proc. Natl Acad. Sci. USA 104 2050-5

[15] Chen F, Wang G, Griffin J I, Brenneman B, Banda N K, Holers V M, Backos D S, Wu L, Moghimi S M and Simberg D 2017 Complement proteins bind to nanoparticle protein corona and undergo dynamic exchange in vivo Nat Nano $12387-93$

[16] Hu C-M J et al 2015 Nanoparticle biointerfacing via platelet membrane cloaking Nature 526 118-21

[17] Zhang Y, You B, Hsu W, Ren C, Li X and Wang J 2015 Silica based nanocapsules: synthesis, structure control and biomedical applications Chem. Soc. Rev. 44315-35

[18] Wang F, Yu L, Monopoli M P, Sandin P, Mahon E, Salvati A and Dawson K A 2013 The biomolecular corona is retained during nanoparticle uptake and protects the cells from the damage induced by cationic nanoparticles until degraded in the lysosomes Nanomedicine: Nanotechnology, Biology and Medicine 9 1159-68

[19] Maiolo D, Del Pino P, Metrangolo P, Parak W J and Bombelli F B 2015 Nanomedicine delivery: does protein corona route to the target or off road? Nanomedicine $103231-47$

[20] Bekdemir A and Stellacci F 2016 A centrifugation-based physicochemical characterization method for the interaction between proteins and nanoparticles Nat. Commun. 71

[21] Wang L, Li J, Pan J, Jiang X, Ji Y, Li Y, Qu Y, Zhao Y, Wu X and Chen C 2013 Revealing the binding structure of the protein corona on gold nanorods using synchrotron radiation-based techniques: understanding the reduced damage in cell membranes J. Am. Chem. Soc. 135 17359-68

[22] Saptarshi S R, Duschl A and Lopata A L 2013 Interaction of nanoparticles with proteins: relation to bio-reactivity of the nanoparticle Journal of Nanobiotechnology 1126

[23] Flores C Y, Achilli E and Grasselli M 2016 Radiation-induced preparation of core/shell gold/albumin nanoparticles Radiat. Phys. Chem. 142 60-4

[24] Frens G 1973 Controlled nucleation for the regulation of the particle size in monodisperse gold suspensions Nature Physical Science 24 20-2

[25] Mendoza-Herrera L J, Munetón Arboleda D, Schinca D C and Scaffardi L B 2014 Determination of plasma frequency, damping constant, and size distribution from the complex dielectric function of noble metal nanoparticles J. Appl. Phys. 116233105

[26] Mendoza-Herrera L J, Scaffardi L B and Schinca D C 2016 High spectral field enhancement and tunability in core-double shell metaldielectric-metal spherical nanoparticles RSCAdv. 6110471-81

[27] Pfeiffer C, Rehbock C, Huhn D, Carrillo-Carrion C, Jimenez de Aberasturi D, Merk V, Barcikowski S and Parak W J 2014 Interaction of colloidal nanoparticles with their local environment: the (ionic) nanoenvironment around nanoparticles is different from bulk and determines the physico-chemical properties of the nanoparticles Journal of The Royal Society Interface 1120130931

[28] Chen Y, Xianyu Y and Jiang X 2017 Surface modification of gold nanoparticles with small molecules for biochemical analysis Acc. Chem. Res. 50 310-9

[29] Ghosh P, Han G, De M, KyuKim C and Rotello V M 2008 Gold nanoparticles in delivery applications Adv. Drug. Deliv. Rev. 60 1307-15

[30] Zeng S, Yong K-T, Roy I, Dinh X-Q, Yu X and Luan F 2011 A review on functionalized gold nanoparticles for biosensing applications Plasmonics 6491

[31] Syedmoradi L, Daneshpour M, Alvandipour M, Gomez F A, Hajghassem H and Omidfar K 2017 Point of care testing: the impact of nanotechnology Biosensors and Bioelectronics $87373-87$

[32] Chen Y-M, Yu C-J, Cheng T-L and Tseng W-L 2008 Colorimetric detection of lysozyme based on electrostatic interaction with human serum albumin-modified gold nanoparticles Langmuir 243654-60

[33] López-Viota J, Mandal S, Delgado A V, Luis Toca-Herrera J, Moller M, Zanuttin F, Balestrino M and Krol S 2009 Electrophoretic characterization of gold nanoparticles functionalized with human serum albumin (hsa) and creatine J. Colloid Interface Sci. 332 215-23

[34] Bohidar H B 1989 Light scattering study of solution properties of bovine serum albumin, insulin, and polystyrene under moderate pressure Colloid and Polymer Science 267 292-300

[35] Huang S 2002 Optical spectroscopic studies of the dispersibility of gold nanoparticle solutions J. Appl. Phys. 92 7486-90

[36] Pérez Osney O, Díaz Rodríguez J, Zumalacárregui L and Gozá León O 2010 Evaluation of physical properties of ethanolwater mixtures (II). Revista Facultad de Ingeniería Universidad de Antioquia 52 62-74

[37] Tsai D-H, DelRio F W, Keene A M, Tyner K M, MacCuspie R I, Cho T J, Zachariah M R and Hackley V A 2011 Adsorption and conformation of serum albumin protein on gold nanoparticles investigated using dimensional measurements and in situ spectroscopic methods Langmuir 27 2464-77

[38] Achilli E, Casajus G, Siri M, Flores C, Kadlubowskic S, del S, Alonso V and Grasselli M 2015 Preparation of protein nanoparticle by dynamic aggregation and ionizing-induced crosslinking Colloids and Surfaces A: Physicochemical and Engineering Aspects 486 161-71

[39] Kratz F 2008 Albumin as a drug carrier: design of prodrugs, drug conjugates and nanoparticles Journal of Controlled Release 132 171-83

[40] Elzoghby A O, SamyNazik W M and Elgindy A 2012 Albumin-based nanoparticles as potential controlled release drug delivery systems Journal of Controlled Release 157 168-82

[41] Soto Espinoza S L, Sánchez M L, Risso V, Smolko E E and Grasselli M 2012 Radiation synthesis of seroalbumin nanoparticles Radiat. Phys. Chem. 81 1417-21

[42] Hiroki A, Pimblott S M and LaVerne J A 2002 Hydrogen peroxide production in the radiolysis of water with high radical scavenger concentrations The Journal of Physical Chemistry A 106 9352-8

[43] Queiroz R G, Varca G H C, Kadlubowski S, Ulanski P and Lugao A B 2016 Radiation-synthesized protein-based drug carriers: sizecontrolled bsa nanoparticles International Journal of Biological Macromolecules 85 82-91 